\title{
Nucleosome positioning is unaltered at MLH1 splice site mutations in cells derived from Lynch syndrome patients
}

\author{
Mathew A Sloane ${ }^{1}$, Luke B Hesson ${ }^{1}$, Andrea C Nunez ${ }^{1}$, Bryony A Thompson ${ }^{2}$ and Robyn L Ward ${ }^{1 *}$
}

\begin{abstract}
Background: Splicing is more efficient when coupled with transcription and it has been proposed that nucleosomes enriched in exons are important for splice site recognition. Lynch syndrome is a familial cancer syndrome that can be caused by the autosomal dominant inheritance of splice site mutations in the MutL homolog 1 (MLH1) gene. To better understand the role of nucleosomes in splicing, we used MLH1 splice site mutations in Lynch syndrome cases as a model to investigate if abnormal splicing was associated with altered nucleosome positioning at exon-intron boundaries.
\end{abstract}

Findings: Nucleosome Occupancy and Methylome sequencing (NOMe-seq) was used to determine the allele-specific positioning of nucleosomes around heterozygous splice site mutations in lymphoblastoid cells lines (LCLs) derived from six Lynch syndrome patients. These mutations were previously shown to cause exon skipping in five of the six patients. Allele-specific high-resolution nucleosome mapping across exons and exon-intron boundaries revealed high levels of nucleosomes across all regions examined. Alleles containing donor or acceptor splice site mutations showed no consistent alteration in nucleosome positioning or occupancy.

Conclusion: Nucleosomes were enriched at MLH1 exons in LCLs derived from Lynch syndrome patients, and in this model system the positioning of nucleosomes was unaltered at exon-intron boundaries containing splice site mutations. Thus, these splice site mutations alone do not significantly change the local organisation of nucleosomes.

Keywords: Lynch syndrome, Colorectal cancer, Nucleosome, Splice site, Splicing, Acceptor, Donor, Exon

\section{Findings \\ Background}

Splicing removes introns from a longer pre-cursor RNA molecule to produce a final processed mRNA. Splice donor and acceptor sites located at the junctions between exons and introns contain conserved sequence elements that are necessary for splicing [1]. Although in vitro splicing can occur, splicing is more efficient when coupled with transcription [2], suggesting that factors additional to the nucleic acid sequence are important for splice site recognition. It has been proposed that the positioning of nucleosomes at exons aids in splice-site recognition $[3,4]$.

\footnotetext{
* Correspondence: robyn@unsw.edu.au

${ }^{1}$ Adult Cancer Program, Lowy Cancer Research Centre and Prince of Wales Clinical School, University of New South Wales, Sydney, NSW, Australia Full list of author information is available at the end of the article
}

Nucleosome positioning describes the precise location of a given nucleosome, whereas nucleosome occupancy refers to the proportion of molecules bearing a nucleosome at a specific location, at any given instant [5]. It is hypothesised that nucleosomes positioned within exons, especially those with weak splice sites, cause RNA Polymerase II (RNAPII) to pause, enabling an interaction with the spliceosome and more efficient splicing [3]. In support of this, nucleosome occupancy is enriched across exons $[3,4]$, which have a high GC content that favours nucleosome assembly $[3,6]$, and the average size of an exon within the body of a human gene is $151 \mathrm{bp}$, that is similar in length to nucleosomal DNA (approximately $147 \mathrm{bp}$ ) [7]. Splicing factors associate with the C-terminal domain of RNA Polymerase II (RNAPII) [8], while the histone modification H3K36me3 is enriched in exons $[3,9]$, where it may act as a scaffold to recruit splicing factors [9]. Taken together this indicates there is 
interplay between nucleosomes, the splicing machinery and the DNA sequence.

Lynch syndrome is an autosomal dominant familial cancer syndrome characterised by early onset colorectal, endometrial and other cancers $[10,11]$. It is most commonly caused by the inheritance of heterozygous loss-offunction mutations, including splice site mutations, in the DNA mismatch repair (MMR) genes $M L H 1$ and $M S H 2$ [12]. In this study we used $M L H 1$ splice site mutations in Lynch syndrome as a model to better understand the role of nucleosomes in splicing. We investigated cells from Lynch syndrome patients with splice site mutations to determine whether splicing aberrations were associated with altered nucleosome positioning at exon-intron boundaries.

\section{Materials and methods}

\section{Identification of $M L H 1$ splice site mutations}

Bioinformatic analysis and in vitro assays previously showed that genetic mutations at exon-intron boundaries in the $M L H 1$ gene generate aberrantly spliced transcripts (see Table 1). All mutations were classified as pathogenic (Class 5) according to the International Society for Gastrointestinal Hereditary Tumours Variant Interpretation Committee (InSiGHT VIC) [12,13].

\section{Cell culture}

LCLs were established from patient blood by transformation with Epstein-Barr Virus (as described previously) [13], and cultured in RPMI with $10 \%$ fetal bovine serum (Gibco, Life Technologies) at $37^{\circ} \mathrm{C}$ in $5 \% \mathrm{CO}_{2}$. Lymphocytes for transformation from five patients (c.588 + $1 \mathrm{G}>\mathrm{T}$, c.589-2A > G, c.791-1G > C, c.884G > A and c.1559-2A > T) were obtained from the Australasian Colorectal Cancer Family Registry (ACCFR) [14]. Lymphocytes from the patient with the c.790 + 1G > T mutation were obtained from the MCO collection [15].

\section{NOMe-seq}

NOMe-seq was performed as described previously [16]. Briefly, intact nuclei were treated with 200 to $300 \mathrm{U}$ $\mathrm{GpC}$ methyltransferase M.CviPl and 160 to $320 \mu \mathrm{M}$ $\mathrm{S}$-adenosylmethionine for $15 \mathrm{~min}$ at $37^{\circ} \mathrm{C}$ followed by termination of the reaction with an equal volume of $20 \mathrm{mM}$ Tris $\mathrm{HCl} \mathrm{pH} 7.9,600 \mathrm{mM} \mathrm{NaCl}, 1 \%$ (w/v) SDS and $10 \mathrm{mM}$ EDTA. DNA was extracted using phenol chloroform followed by ethanol precipitation and bisulfite modified using the EZ DNA Methylation-Gold ${ }^{\text {tw }}$ Kit (Zymo Research). Regions incorporating the splice site mutation in each patient were amplified from $40 \mathrm{ng}$ of bisulfite treated DNA using a nested PCR with the primers and annealing temperatures described in Table 2. Single molecule sequencing of PCR amplicons was performed as described previously [17]. Wild-type and mutant alleles were distinguished using the splice site sequence alteration. The M.CviPI enzyme methylates accessible DNA at GpC sites, whereas nucleosome bound DNA is inaccessible and remains refractory to $\mathrm{GpC}$ methylation. Regions of M.CviPI inaccessibility of $\geq 150 \mathrm{bp}$ (the length of DNA wrapped around a single nucleosome) within a single molecule were considered to represent regions of nucleosome occupancy. In addition, NOMe-seq retains the endogenous methylation status of the DNA allowing nucleosome positions and DNA methylation to be determined on each molecule.

\section{Results}

We determined the positioning of nucleosomes across exons 7, 8, 9, 10 or 14 of the $M L H 1$ gene in LCLs derived

Table 1 Molecular features of MLH1 splice site mutations in six individuals with Lynch syndrome

\begin{tabular}{|c|c|c|c|c|c|}
\hline \multirow[t]{2}{*}{ Mutation } & \multirow{2}{*}{$\begin{array}{l}\text { Mutation location } \\
\text { (GRC37, Feb 2009) }\end{array}$} & \multirow{2}{*}{$\begin{array}{l}\text { Affected } \\
\text { splice site }\end{array}$} & \multicolumn{2}{|c|}{ Nucleosome occlusion of splice site } & \multirow[t]{2}{*}{ Splicing error [13] } \\
\hline & & & Allele & Proportion of alleles & \\
\hline \multirow[t]{3}{*}{$c .588+1 G>T$} & Chr. 3: $37,053,354$ & Donor & Wild-type (G) & $19 / 20$ & \multirow[t]{3}{*}{ Exon 7 skipping (r.546_588del) } \\
\hline & \multirow[t]{2}{*}{ First bp of intron 7} & & Mutant (T) & $21 / 22$ & \\
\hline & & Acceptor & Homozygous & $36 / 42$ & \\
\hline \multirow[t]{2}{*}{ c. $.589-2 A>G$} & Chr. 3: $37,053,500$ & Acceptor & Wild-type (A) & $17 / 20$ & \multirow[t]{2}{*}{4 bp deletion in cDNA (r.589_592del) } \\
\hline & 2nd last bp of intron 7 & & Mutant (G) & $19 / 22$ & \\
\hline \multirow[t]{2}{*}{ c. $790+1 G>T$} & Chr. 3: $37,056,036$ & Donor & Wild-type (G) & $33 / 34$ & \multirow[t]{2}{*}{ Unknown } \\
\hline & 1st bp of intron 9 & & Mutant (T) & $21 / 24$ & \\
\hline \multirow[t]{2}{*}{ c.791-1G >C } & Chr. 3: $37,058,996$ & Acceptor & Wild-type (G) & $30 / 30$ & \multirow[t]{2}{*}{ Exon 10 skipping (r.791_884del) } \\
\hline & Last bp of intron 9 & & Mutant (C) & $12 / 18$ & \\
\hline \multirow[t]{2}{*}{$c .884 G>A$} & Chr. 3: $37,059,090$ & Donor & Wild-type (G) & $42 / 42$ & \multirow[t]{2}{*}{ Exon 10 skipping (r.791_884del) } \\
\hline & Last bp of exon 10 & & Mutant (A) & $24 / 30$ & \\
\hline \multirow[t]{2}{*}{ c.1559-2A > T } & Chr. 3: $37,081,675$ & Acceptor & Wild-type (A) & $17 / 20$ & \multirow{2}{*}{$\begin{array}{l}\text { Two aberrant transcripts (Exon } 14 \text { skipping o } \\
\text { exon } 14 \text { and } 15 \text { skipping - r.[1559_1667del, } \\
\text { 1559_1731del]) }\end{array}$} \\
\hline & Second last bp of intron 13 & & Mutant (T) & $16 / 16$ & \\
\hline
\end{tabular}


Table 2 NOMe-seq primer sequences and amplification conditions

\begin{tabular}{|c|c|c|}
\hline Region & NOMe-seq primers & Annealing $\left({ }^{\circ} \mathrm{C}\right)$ \\
\hline \multirow[t]{3}{*}{$\mathrm{c.} .588+1 \mathrm{G}>\mathrm{T}$} & F:TTGATATTTAGTGTGTGTTITGGGYAT & $\mathrm{F} / \mathrm{R}=54^{\circ} \mathrm{C}$ \\
\hline & R:CACATAATATCTTAAAAAATTCCAAAATAATA & $\mathrm{F} / \mathrm{RN}=56^{\circ} \mathrm{C}$ \\
\hline & RN:ATACCRACTAACARCATTTCCAAAAATAA & \\
\hline \multirow[t]{3}{*}{ c. $589-2 \mathrm{~A}>\mathrm{G}$} & F:TTAGGTATTTAGTATATAATGYAGG & $\mathrm{F} / \mathrm{R}=51^{\circ} \mathrm{C}$ \\
\hline & R:CACTATAAATATTITCAAAACTAAAACCTTA & $\mathrm{F} / \mathrm{RN}=52^{\circ} \mathrm{C}$ \\
\hline & RN:CACAAAATCTAAAAAATTACATACACCTAA & \\
\hline \multirow[t]{3}{*}{$c .790+1 G>T$} & F:TAGGYATAGGAGGATTITAAATGGATTAAGTT & $\mathrm{F} / \mathrm{R}=52^{\circ} \mathrm{C}$ \\
\hline & R:CAATTTCTTTAATAACAATRCCTATACCTAAA & $\mathrm{F} / \mathrm{RN}=52^{\circ} \mathrm{C}$ \\
\hline & RN:TTACTCRTAAAAACTCTAACACCATCAA & \\
\hline \multirow[t]{3}{*}{ c.791-1G >C } & F:GATGTGATGTGYATATTATTATAGAAATGTT & $\mathrm{F} / \mathrm{R}=55^{\circ} \mathrm{C}$ \\
\hline & R:TATCARCACCTCCTAATAAAATGAARCATA & $\mathrm{F} / \mathrm{RN}=56^{\circ} \mathrm{C}$ \\
\hline & RN:ATCCTTTTRCCAATAATATATAAAATTCACTCTA & \\
\hline \multirow[t]{3}{*}{ c. $884 \mathrm{G}>\mathrm{A}$} & F:GGATGTGATGTGYATATTATTATAGAAATGTT & $\mathrm{F} / \mathrm{R}=58^{\circ} \mathrm{C}$ \\
\hline & R:CTATTATARCTTCCCAACTAACCCCARCAA & $\mathrm{F} / \mathrm{RN}=58^{\circ} \mathrm{C}$ \\
\hline & RN:CTACAARCTATCARCACCTCCTAATAA & \\
\hline \multirow[t]{3}{*}{ c. $1559-2 A>T$} & F:TATTAGGAGGYTTAATTTAGGYTIITTGGTTAT & $\mathrm{F} / \mathrm{R}=58^{\circ} \mathrm{C}$ \\
\hline & R:ACCCTCACCACCTAATTCACAACATTTATAA & $\mathrm{F} / \mathrm{RN}=57^{\circ} \mathrm{C}$ \\
\hline & RN:ACTAARCAACTACCAAAAACTAARCTTCTTA & \\
\hline \multirow[t]{2}{*}{ HSPA5 } & F: GAGAAGAAAAAGTTTAGATTTATA & $\mathrm{F} / \mathrm{R}=56^{\circ} \mathrm{C}$ \\
\hline & R: AAACACCCCAATAAATCAATC & \\
\hline
\end{tabular}

from six Lynch syndrome patients. Splice site mutations within or near these exons affected splice donor or acceptor sites (Table 1). NOMe-seq and single molecule sequencing allowed us to distinguish mutant from wildtype alleles using the splice site sequence mutation. At all sites analysed the majority of molecules were inaccessible at $\mathrm{GpC}$ sites and methylated at $\mathrm{CpG}$ sites (Figure 1). NOMe-seq analysis of the control gene HSPA5, a constitutively active gene that maintains a nucleosome-depleted region at the transcription start site [18], showed that the CpG island was unmethylated (data not shown) and confirmed that $\mathrm{GpC}$ inaccessibility was due to nucleosome occupancy rather than incomplete M.CviPI treatment (Figure 2). This shows that the majority of molecules at the sites analysed are occupied by nucleosomes in these cells.

Separation of alleles by presence or absence of the splice site mutation showed no overall difference in either nucleosome positioning or occupancy between wild-type and mutant alleles (Figure 1 and Table 1). In the six splice site mutations analysed, three were located within donor splice sites and three were located within acceptor splice sites (Table 1). Though some difference in precise positioning between mutant and wild-type alleles was observed across some exons (for example, exons 7 and 10, Figure 1B) this was confined to a small subset of molecules, rather than the majority of molecules as would be expected if the mutations affected nucleosome positioning. Our study therefore shows that nucleosome occupancy is unaltered at exon-intron boundaries containing these splice site mutations.

\section{Discussion}

In this study, we show that nucleosome positioning is unaltered at exon-intron boundaries containing splice site mutations. This finding suggests that the positioning of nucleosomes at exon-intron boundaries containing splice site mutations does not play a role in the splicing defect.

NOMe-seq has been used to measure nucleosome occupancy at gene promoters, CTCF binding sites [19] and regulatory elements [20] but this is the first study that has utilised the technique to measure nucleosome occupancy at splice sites. NOMe-seq previously showed that the active CpG island (CGI) promoter of HSPA5 has a nucleosome-depleted region (NDR) upstream of the transcriptional start site [19]. This region was included as a control in each assay, and in all cases the NDR was present. This indicated that the absence of $\mathrm{GpC}$ methylation observed in $M L H 1$ was caused by nucleosome occlusion, not an artefact caused by a defective $M$. CviPI enzyme. In addition, CpG methylation was absent from the HSPA5 NDR which is a feature of active CGI promoters [21]. Finally, gene body methylation is a feature 







\begin{tabular}{|c|c|c|}
\hline \multicolumn{3}{|c|}{150 bp } \\
\hline \multicolumn{3}{|l|}{ B } \\
\hline 1. c. $588+1 G>T$ & 2. c.589-2A>G & 3. c. $790+1 G>T$ \\
\hline 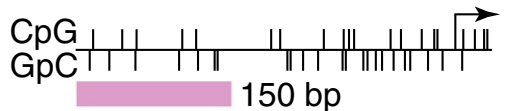 & 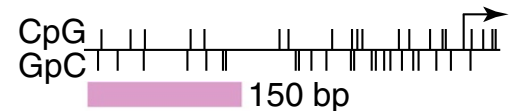 & 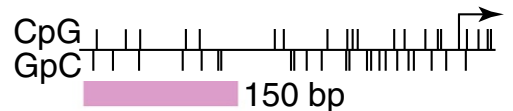 \\
\hline 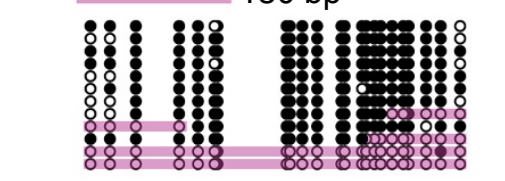 & 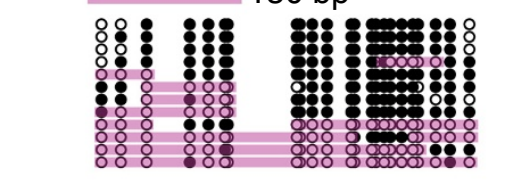 & 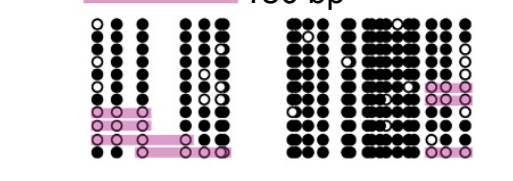 \\
\hline 4. c. $791-1 G>C$ & 5. c. $844 \mathrm{G}>\mathrm{A}$ & 6. c. $1559-2 A>T$ \\
\hline 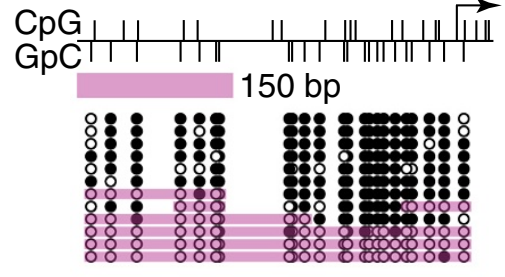 & 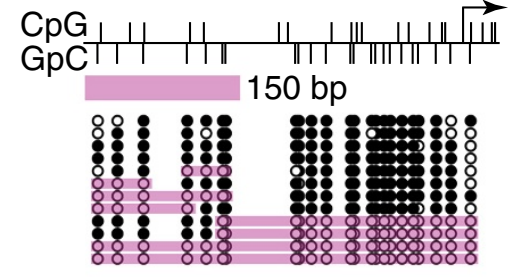 & 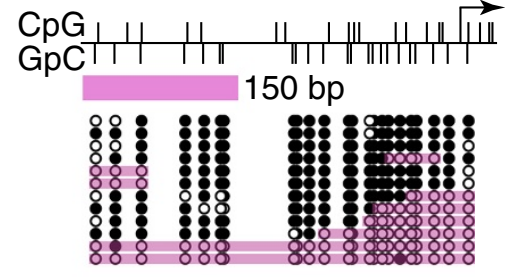 \\
\hline $\begin{array}{l}\text { Legend: } \\
\text { Methylated GpC (ac } \\
\text { Unmethylated GpC } \\
\text { Nucleosome }\end{array}$ & $\begin{array}{l}\text { sible) } \\
\text { ccessible) }\end{array}$ & \\
\hline $\begin{array}{l}\text { Figure } 2 \text { Nucleosome occupancy and end } \\
\text { NOMe-seq assay (black bar) in relation to the } \\
\text { the position of nucleosomes on individual D } \\
\text { in MLH1. Vertical black bars below line = GpC } \\
\text { DNA molecules as determined by GpC methylt } \\
\text { GpC/inaccessible to M.CViPI. All endogenous Cp }\end{array}$ & $\begin{array}{l}\text { us CPG methylation at the HSPA5 gene. ( } \\
\text { promoter. The arrow represents the transcrip } \\
\text { lecules at the HSPA5 gene in } L C L s \text { derived fro } \\
\text { vertical black bars above line }=C p G \text { sites; pink } \\
\text { ase inaccessibility; black circles = methylated Gp } \\
\text { were unmethylated and triangles are not show }\end{array}$ & $\begin{array}{l}\text { chematic showing the location of the } \\
\text { nal start site. (B) NOMe-seq was used to map } \\
\text { he six individuals with splice site mutations } \\
\mathrm{s}=\text { the location of nucleosomes on individual } \\
\text { cessible to M.CviPl; white circles = unmethylated } \\
\text { b provide better clarity of GpC sites. }\end{array}$ \\
\hline
\end{tabular}

of human cells [22,23] and the majority of CpG sites within the MLH1 exons and introns were methylated. Together, these controls indicated that the NOMe-seq assays captured the true state of nucleosome positioning at $M L H 1$ splice sites.

Alternative splicing of $M L H 1$ occurs in a range of normal tissues [24], but several pieces of evidence indicate that the transcripts observed in our study are caused by the mutation, rather than being normal splicing events. First, the splicing aberrations were predicted with bioinformatic splicing software [13], and second, although one of the aberrant transcripts $(\Delta 10)$ has been reported among naturally occurring $M L H 1$ splice transcripts, the clinical data associated with these variants are also indicative of pathogenicity as demonstrated by the results of previous multifactorial likelihood analyses [13].

One strength of our study was the ability to determine the allele-specific position of nucleosomes by incorporating a heterozygous single nucleotide variant into each NOMe-seq assay. Small changes in positioning were observed but these molecules constituted approximately the same small proportion of total molecules on both alleles. Subtle changes in nucleosome positioning have been considered important in regulating the expression of cell cycle-dependent genes [18] and enhancer accessibility [25], but in those studies the subtle changes were observed on a significant number of molecules that resulted in an overall change in nucleosome occupancy. 
There was no consistent alteration in nucleosome occupancy between the wild-type and mutant alleles at a variety of donor and acceptor sites, which would be expected if nucleosome positioning at these sites played a role in mediating the effects of splice site mutations. Together our data shows that alleles containing a splice site mutation show no differences in nucleosome occupancy to wild-type alleles.

A limitation of the approach was that in most of the assays (assays 2 to 6 ) it was only possible to measure nucleosome positions at either the donor site or the acceptor site. Bisulfite conversion causes fragmentation of genomic DNA and it is technically challenging to amplify fragments greater than $500 \mathrm{bp}$ in length [26]. The donor and acceptor sites were separated by more than $2 \mathrm{~kb}$ of intronic sequence, making it impossible to concurrently determine the allele-specific position of nucleosomes at the donor and acceptor site on the same molecule. Although an independent NOMe-seq assay could be performed at the other donor or acceptor site, it would provide no information on allele-specificity. The data from one assay (c.588 + $1 \mathrm{G}>\mathrm{T}$ ), however, in which both splice sites were present in the one amplicon, showed no significant change in nucleosome occupancy at the donor or acceptor site in intron 7.

Previous studies have utilised genome-wide datasets of micrococcal nuclease (MNase) digested chromatin to investigate nucleosome occupancy $[3,4]$. Here we used NOMe-seq to map the position of nucleosomes relative to exons and splice sites in a single gene at single molecule resolution. This approach provides the most accurate possible measurement of nucleosome positioning and enables allele-specific mapping of nucleosomes. In agreement with previous reports in humans [3,4], Caenorhabditis elegans [3,4] and Drosophila melanogaster [3], we observed high nucleosome occupancy at exons. Thus, the findings from genome-wide studies were supported by our independent, single-molecule approach at the $M L H 1$ gene.

A key finding of our study was that nucleosome positioning was not significantly affected by mutations at the MLH1 splice sites investigated. Recent work with an in vitro IKBKAP mini-gene system found that alternative splicing changed chromatin organisation, with splice site strength and factors needed for splicing, such as U1 snSNP, playing a role in the regulation of nucleosome occupancy in exons [27]. This and an earlier study [3] indicate that splice site strength is an important determinant of nucleosome occupancy in exons. Exons with stronger polypyrimidine tracts (PPT; one of the conserved DNA elements located at 3 ' splice sites) have increased nucleosome occupancy compared to immediately adjacent introns, suggesting that nucleosomes act as a barrier that help to define the physical location of the 3 ' splice site [3]. Although we investigated an equal number of donor and acceptor splice site mutations, nucleosome positioning was not significantly different between exons and the immediate $5^{\prime}$ and $3^{\prime}$ intronic sequence on the wild-type or mutant allele. The discrepancy between earlier studies and ours may in part relate to the strength of the $M L H 1$ splice sites investigated. Alternatively, changes to chromatin organisation induced by splice mutations may be different to those caused by normal splicing events. Co-transcriptional splicing involves a complex interplay between RNAPII, the spliceosome [8] and histone modifications [3,9] and alterations in these factors, in addition to DNA sequence changes may be needed to alter nucleosome occupancy at exons.

In summary this study used NOMe-seq to determine the allele-specific position of nucleosomes in relation to MLH1 splice site mutations. We conclude that splice site mutations that cause aberrant splicing of $M L H 1$ do not alone significantly affect local nucleosome positioning in LCLs from Lynch syndrome patients.

\section{Competing interests}

The authors declare that they have no competing interests.

\section{Authors' contributions}

MAS and LBH designed NOM-seq assays. ACN and MAS performed NOMe-seq assays. BAT provided LCL samples. RLW and LBH conceived the study, participated in its coordination and helped to draft the manuscript. All authors read and approved the final manuscript.

\section{Acknowledgements}

RLW is supported by Cancer Council NSW (RG 13-07) and Cancer Australia. We would like to thank the Australasian Colorectal Cancer Family Registry (ACCFR) for providing lymphocytes for transformation.

\section{Author details}

${ }^{1}$ Adult Cancer Program, Lowy Cancer Research Centre and Prince of Wales Clinical School, University of New South Wales, Sydney, NSW, Australia. ${ }^{2}$ Department of Genetics and Computational Biology, QIMR Berghofer Medical Research Institute, Brisbane, QLD, Australia.

Received: 4 July 2014 Accepted: 28 November 2014

Published online: 13 December 2014

\section{References}

1. Black DL: Mechanisms of alternative pre-messenger RNA splicing. Annu Rev Biochem 2003, 72:291-336.

2. Das R, Dufu K, Romney B, Feldt M, Elenko M, Reed R: Functional coupling of RNAP II transcription to spliceosome assembly. Genes Dev 2006, 20:1100-1109.

3. Schwartz S, Meshorer E, Ast G: Chromatin organization marks exon-intron structure. Nat Struct Mol Biol 2009, 16:990-995.

4. Tilgner H, Nikolaou C, Althammer S, Sammeth M, Beato M, Valcárcel J, Guigó R: Nucleosome positioning as a determinant of exon recognition. Nat Struct Mol Biol 2009, 16:996-1001.

5. Berrozpe G, Bryant GO, Warpinski K, Ptashne M: Regulation of a mammalian gene bearing a CpG island promoter and a distal enhancer. Cell Rep 2013, 4:445-453.

6. Field Y, Kaplan N, Fondufe-Mittendorf Y, Moore IK, Sharon E, Lubling Y, Widom J, Segal E: Distinct modes of regulation by chromatin encoded through nucleosome positioning signals. PLOS Comput Biol 2008, 4:e1000216.

7. Wang Z, Burge CB: Splicing regulation: from a parts list of regulatory elements to an integrated splicing code. RNA 2008, 14:802-813.

8. de Almeida SF, Carmo-Fonseca M: The CTD role in cotranscriptional RNA processing and surveillance. FEBS Lett 2008, 582:1971-1976. 
9. Kolasinska-Zwierz P, Down T, Latorre I, Liu T, Liu XS, Ahringer J: Differential chromatin marking of introns and expressed exons by H3K36me3. Nat Genet 2009, 41:376-381.

10. Watson P, Lynch HT: Extracolonic cancer in hereditary nonpolyposis colorectal cancer. Cancer 1993, 71:677-685.

11. Lynch HT, de la Chapelle A: Genetic susceptibility to non-polyposis colorectal cancer. J Med Genet 1999, 36:801-818.

12. Thompson BA, Spurdle AB, Plazzer JP, Greenblatt MS, Akagi K, Al-Mulla F, Bapat B, Bernstein I, Capellá G, den Dunnen JT, du Sart D, Fabre A, Farrell MP, Farrington SM, Frayling IM, Frebourg T, Goldgar DE, Heinen CD, Holinski-Feder E, Kohonen-Corish M, Robinson KL, Leung SY, Martins A, Moller P, Morak M, Nystrom M, Peltomaki P, Pineda M, Qi M, Ramesar R, et al: Application of a 5-tiered scheme for standardized classification of 2,360 unique mismatch repair gene variants in the InSiGHT locus-specific database. Nat Genet 2014, 46:107-115.

13. Thompson BA, Goldgar DE, Paterson C, Clendenning M, Walters R, Arnold S, Parsons MT, Michael DW, Gallinger S, Haile RW, Hopper JL, Jenkins MA, Lemarchand L, Lindor NM, Newcomb PA, Thibodeau SN, Colon Cancer Family R, Young JP, Buchanan DD, Tavtigian SV, Spurdle AB: A multifactorial likelihood model for MMR gene variant classification incorporating probabilities based on sequence bioinformatics and tumor characteristics: a report from the Colon Cancer Family Registry. Hum Mutat 2013, 34:200-209.

14. Australasian Colorectal Cancer Family Registry [http://www.pedigree.org. au/pedigree-studies/accfr.aspx]

15. MCO study tumour collection [http://researchdata.ands.org.au/mco-studytumour-collection]

16. Wolff EM, Byun HM, Han HF, Sharma S, Nichols PW, Siegmund KD, Yang AS, Jones PA, Liang G: Hypomethylation of a LINE-1 promoter activates an alternate transcript of the MET oncogene in bladders with cancer. PLoS Genet 2010, 6:e1000917.

17. Hesson $L B$, Ward RL: Discrimination of pseudogene and parental gene DNA methylation using allelic bisulfite sequencing. Methods $\mathrm{Mol}$ Biol 2014, 1167:265-274.

18. Kelly TK, Miranda TB, Liang G, Berman BP, Lin JC, Tanay A, Jones PAPA: H2A. $\mathrm{Z}$ maintenance during mitosis reveals nucleosome shifting on mitotically silenced genes. Mol Cell 2010, 39:901-911.

19. Kelly TK, Liu Y, Lay FD, Liang G, Berman BP, Jones PA: Genome-wide mapping of nucleosome positioning and DNA methylation within individual DNA molecules. Genome Res 2012, 22:2497-2506.

20. Taberlay PC, Statham AL, Kelly TK, Clark SJ, Jones PA: Reconfiguration of nucleosome depleted regions at distal regulatory elements accompanies DNA methylation of enhancers and insulators in cancer. Genome Res 2014, Epub ahead of print.

21. Deaton $A M, B i r d A: C p G$ islands and the regulation of transcription. Genes Dev 2011, 25:1010-1022.

22. Ball MP, Li JB, Gao Y, Lee JH, LeProust EM, Park IH, Xie B, Daley GQ, Church GM: Targeted and genome-scale strategies reveal gene-body methylation signatures in human cells. Nat Biotechnol 2009, 27:361-368.

23. Chodavarapu RK, Feng S, Bernatavichute YV, Chen PY, Stroud H, Yu Y, Hetzel JA, Kuo F, Kim J, Cokus SJ, Casero D, Bernal M, Huijser P, Clark AT, Krämer U, Merchant SS, Zhang X, Jacobsen SE, Pellegrini M: Relationship between nucleosome positioning and DNA methylation. Nature 2010, 466:388-392.

24. Thompson BA, Martins A, Spurdle AB: A review of mismatch repair gene transcripts: issues for interpretation of mRNA splicing assays. Clin Genet 2014. Epub ahead of print.

25. Andreu-Vieyra C, Lai J, Berman BP, Frenkel B, Jia L, Jones PA, Coetzee GA: Dynamic nucleosome-depleted regions at androgen receptor enhancers in the absence of ligand in prostate cancer cells. Mol Cell Bio 2011, 31:4648-4662.

26. Grunau C, Clark SJ, Rosenthal A: Bisulfite genomic sequencing: systematic investigation of critical experimental parameters. Nucleic Acids Res 2001, 29:E65-5.

27. Keren-Shaul H, Lev-Maor G, Ast G: Pre-mRNA splicing is a determinant of nucleosome organization. PLoS One 2013, 8:e53506.

\section{doi:10.1186/s13148-014-0032-6}

Cite this article as: Sloane et al: Nucleosome positioning is unaltered at MLH1 splice site mutations in cells derived from Lynch syndrome patients. Clinical Epigenetics 2014 6:32.

\section{Submit your next manuscript to BioMed Central and take full advantage of:}

- Convenient online submission

- Thorough peer review

- No space constraints or color figure charges

- Immediate publication on acceptance

- Inclusion in PubMed, CAS, Scopus and Google Scholar

- Research which is freely available for redistribution 